Applied Physics A manuscript No.

(will be inserted by the editor)

\title{
Mixed-mode crack tip loading and crack deflection in 1D quasicrystals
}

\author{
Zhibin Wang, Johannes Scheel · Andreas Ricoeur
}

Received: date / Accepted: date

Abstract Quasicrystals (QC) are a new class of materials besides crystals and amorphous solids and have aroused much attention of researchers since they were discovered. This paper presents a generalized fracture theory including the J-integral and crack closure integrals, relations between $J_{1}, J_{2}$ and the stress intensity factors as well as the implementation of the near-tip stress and displacement solutions of 1D QC. Different crack deflection criteria, i.e. the J-integral and maximum circumferential stress criteria are investigated for mixed-mode loading conditions accounting for phononphason coupling. One focus is on the influence of phason stress intensity factors on crack deflection angles.

Keywords quasicrystal $\cdot$ crack deflection $\cdot$ mixed-mode loading $\cdot$ fracture quantities $\cdot$ J-integral

\section{Introduction}

In 1982 D. Shechtman observed in the diffraction patterns from electronic microscopy that an $\mathrm{Al}_{86} \mathrm{Mn}_{14}$ alloy abnormally exhibits a five-fold orientational symmetry and published the result two years later [1], motivating great discussion [2]. The crystallographic community agreed that five-fold symmetry is disallowed in crystalline structures, which means this alloy is a new class of material besides crystals and amorphous solids. On the other side, researchers have mathematically developed incommensurate structures based on

Zhibin Wang

Institute of Mechanics, Department of Mechanical Engineering University of Kassel

Mönchebergstraße 7, 34125 Kassel, Germany

Tel.: +49-561-8043755

Fax: +49-561-8042720

E-mail: zhibin.wang@uni-kassel.de icosahedral lattices and have succeeded in computing the diffraction pattern analytically, thus proving the possibility of their existence [3, 4]. This new material has long-range orientational order but without translational symmetry in particular directions. Lacking translational symmetry, the atom arrangements are quasiperiodic rather than periodic. In short order, however, the structure still has crystalline features, so it was termed as quasicrystal (QC) [3]. Loading a QC structure mechanically, classical displacements are observed, which are denoted as phonon displacements. Additionally, the quasiperiodic arrangement is influenced going along with atomic flips, denoted as phason displacements.

Depending on in how many directions the atom arrangement is quasiperiodic, QCs are categorised into three subclasses, i.e. 1D, 2D and 3D. A QC with one quasiperiodic and two periodic atom arrangement directions, e.g. is called $1 \mathrm{D}$ QC [5]. The QCs found in early years, however, were not thermodynamically stable but just metastable, forming a crystalline structure when be heated. After several years the discovery of QCs being stable up to melting temperature was published [6, 7]. Stable QCs can be found not only in the laboratory but also in nature, although there remain open questions like how these natural QCs are formed [8]. So far only a few of the approximately 50 stable QC structures have been studied quantitatively [9].

The physical properties of QCs are also investigated massively, like thermodynamics, piezoelectricity, light propagation, electron transport, magnetic, conduction, elastic properties, dislocation mechanisms and so on [10,11, 12, 13, 14]. The progress of these investigations extends essentially the fields of application of QC, for instance, wear resistant coating, hydrogen storage devices or sintering powders for rapid prototyping. 
Based on the generalized elastic theory, some researchers have begun to investigate defect and crack behaviour in QCs. Similar to other coupled problems, as a result of inserting a phason field, the conventional fracture and crack deflection criteria may not be suitable for QC. To understand the effect of cracks in QCs and how the coupled phason field influences the crack growth behaviour, an extended fracture theory is prerequisite including the determination of crack tip loading quantities, e.g. stress intensity factors, the strain energy release rate or the J-integral, the knowledge of their mutual dependences and mixed-mode criteria for crack growth and deflection. In particular the latter aspect might be interesting. From classical linear elastic fracture mechanics it is known that small contributions of a Mode-II loading are responsible for curved crack paths. Thus, it might be expected that even a small coupling-induced phason crack tip loading gives rise to crack curvature.

A variety of papers has been published during the past 15 years, dealing with many aspects of the extended theory of elasticity for QC. Various boundary value problems have been solved applying different methods, providing solutions for e.g. inclusions [15, 16], interfaces [17] or Green's functions [18,19]. Some papers deal with crack problems in QC giving solutions for elastic fields in the structure or in the crack tip near field and providing fracture mechanical loading quantities [20,21]. Finite element (FE) approaches have been established in [22,23]. The problem of crack growth in QC has lately been treated by a molecular dynamics approach [24]. To our best knowledge, the problem of crack growth and deflection in QC has, however, not yet been handled within a continuum mechanical framework. The same holds for equations, relating energetical and stress based crack tip loading quantities, i.e. J-integral, energy release rate and stress intensity factors.

The focus of this work is on cracks in 1D quasicrystalline plates. The generalized elastic framework and some fracture mechanical basics of QCs are given in the next two sections. In particular, the relation between the coordinates of the J-integral vector and the stress intensity factors will be drawn from the generalized crack closure integral and configurational forces are derived starting from an energy balance in QC. Then, two crack deflection criteria for QCs under mixed-mode loading are investigated. In the last part, some computed results with different phonon-phason coupling constants and loading regimes are presented and their impact on crack deflection angles is discussed.
2 Theoretical framework of linear elasticity in quasicrystals

\subsection{Phonon and phason fields}

In order to generalize the crystallographic theory and construct the quasicrystalline structure mathematically, the so-called cut method from a higher-dimensional space is introduced [25,26]. Briefly, the reason is that due to their aperiodicity QCs need more than three basis vectors to describe their structures, while for crystals three unit vectors are sufficient. In fact, the periodicity exists in the hyper space which is spanned by the 4 to 6 basis vectors of QCs depending on the number of QC directions. The cut method allows the construction of quasicrystals being cut or projected from this mathematical hyper space onto the 3-dimensional physical space, in which the objects really exist. The additional non-physical dimensions are denoted as complementary or perpendicular space. The symbol $\|$ is used to identify the physical space and $\perp$ stands for the complementary space. For elasticity, the variables from physical space are called phonon and from complementary space phason variables [27].

In order to introduce the cut method intuitively, as well as the phason displacement as a special elementary excitation in complementary space, a simplified model with a 2-dimensional periodic pattern as hyper space is illustrated in Fig. 1. The pattern, represented by the regularly arranged dots, projected onto $e^{\|}$builds a sequence conforming to Fibonacci series as principle quasi-periodic arrangement, if the angle between $e^{\|}$and the periodic direction is irrational. The projected sequence, where S stands for "short" and L for "long", is a real atom or molecule arrangement in QCs, which has been proven by high-resolution transmission electron microscopy [28, 29]. The projection in black describes an initial unloaded state, the other projection in red is the result when an ideal phason strain $\partial W / \partial x^{\|}$is applied, where $x^{\|}$from now on is the coordinate of the physical space and $W$ is the phason displacement in the direction $e^{\perp}$. It is obvious that the arrangement "SLSLLSLLS..." is changed into "SSLSLSLSL..." due to the phason strain. Accordingly, some local combinations exchange their orders like $\mathrm{SL} \longleftrightarrow \mathrm{LS}$, which is denoted as phason flip. Within this context the phason strain is interpreted as rotation $\varphi$ of a sequence, where the two distances ( $\mathrm{S}$ and $\mathrm{L}$ ) keep their lengths, however the arrangement is changed completely, see Fig. 1 for illustration.

According to Landau's theory [30], the mass density function is introduced to describe the statistical position $r_{i}$ which is determined by the probability of 


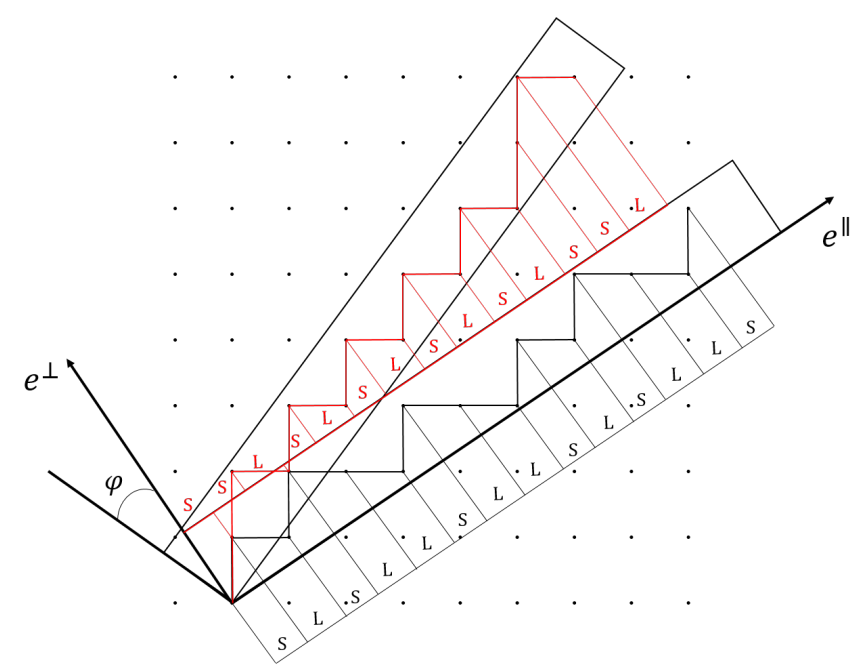

Fig. 1 Cut method to obtain a quasiperiodic arrangement in physical space from a periodic arrangement in hyper space and interpretation of phason strain as rotation $\varphi$ and flips of SLsequences.

the different locations of particles. Generally, the mass density function of a periodic structure is defined as 27]

$\rho\left(r_{i}\right)=\sum_{G_{j}} \rho\left(G_{j}\right) \exp \left\{i G_{j} r_{j}\right\}$,

where $G_{j}$ is the vector of the reciprocal lattice. Here, as in the whole paper, the analytical notation is applied with lowercase indices running from 1 to 3 (1 to 2 in $2 \mathrm{D}$ ) implying summation over double indices. Each $\rho\left(G_{j}\right)$ is a complex order parameter consisting of a magnitude $\rho_{G_{j}}$ and a phase angle $\phi_{G_{j}}$ :

$\rho\left(G_{j}\right)=\rho_{G_{j}} \exp \left\{i \phi_{G_{j}}\right\}$.

The magnitude $\rho_{G_{j}}$ is a state parameter being specific for solid phase matter. It is independent of the position vector $r_{i}$ if the solid is in an unloaded equilibrium state. The phase $\phi_{G_{j}}$ has a basic value $\phi_{G_{j}}^{0}$, indicating an ideal state when all the atoms are at their equilibrium positions. If the atoms are displaced from their equilibrium positions by $u_{j}$ due to mechanical loading or thermodynamic motion, for crystals the phase angle is modified as follows

$\phi_{G_{j}}=\phi_{G_{j}}^{0}+G_{j} u_{j}$

The magnitude, the equilibrium phase and thus the order parameters $\rho\left(G_{j}\right)$ are the factors of a crystalline structure in the sense of Fourier components of periodic order for the reciprocal lattice vector $G_{j}$.

For quasicrystals, where the periodicity exists only in a hyper space consisting of a physical space and a complementary space, the reciprocal lattice $G_{I}(I>3)$ possesses more than 3 dimensions and can be divided into $G_{i}^{\|}$and $G_{i}^{\perp}$. However, the mass density function describes the distribution of mass only in the physical space, i.e.

$\rho\left(r_{i}\right)=\sum_{G_{J}} \rho\left(G_{J}\right) \exp \left\{i G_{j}^{\|} r_{j}\right\}$

As an extension of Eq. (3), the phase angle in the hyper space is given by

$\phi_{G_{I}}=\phi_{G_{I}}^{0}+G_{i}^{\|} u_{i}+G_{j}^{\perp} W_{j}$

where the index $j$ in this equation runs from 1 to $d-3$ with $d$ as the dimension of the hyper space or reciprocal lattice, respectively. $u_{i}$ is the phonon or conventional physical displacement, and $W_{j}$ is the phason displacement. The total displacement $u_{I}=\left(u_{i}^{\|}, u_{i}^{\perp}\right)=\left(u_{i}, W_{i}\right)$ is the shift in the $d$-dimensional space with respect to an equilibrium position. For a $1 \mathrm{D}$ QC, e.g. the total displacement is a vector in a 4-dimensional space, represented by

$u_{I}=u_{i} \vec{e}_{i}^{\|}+W_{1} \vec{e}_{1}^{\perp}$

with the basis vectors $\vec{e}_{i}^{\|}, \vec{e}_{1}^{\perp}$. Generally, the index "1" is omitted in the case of a $1 \mathrm{D}$ QC.

Inserting the phase angle according to Eq. (5) into Eq. (4), the mass density function for quasicrystals is obtained as

$\rho\left(r_{i}\right)=\sum_{G_{J}} \rho_{G_{J}} \exp \left\{i\left(\phi_{G_{J}}^{0}+G_{i}^{\|}\left(r_{i}+u_{i}\right)+G_{j}^{\perp} W_{j}\right)\right\}$.

Within a hydrodynamic theory, the phason displacement can be understood as the local rearrangement of unit cells or particle orders. Details about the phason field and its physical interpretation can be found in diverse review papers [31,32.

Hence, phonon and phason are two kinds of elastic degrees of freedom in QCs. Based on this fact, the generalized theory of elasticity is formulated [30, 33, 34, 35. Since not all directions of a QC body are quasi periodic, $u_{i}$ always consists of three coordinates, while $W_{i}$ may have one $(d=4)$, two $(d=5)$ or three $(d=6)$ according to its quasicrystalline structure. In order to establish a most general theoretical framework of elasticity, 3D QCs are taken into account in Sections 2.2 and 3. Besides that, most of the QCs intrinsically are brittle materials at room temperature, showing extensive ductility just at elevated temperatures [36]. Plastic deformation is thus ignored in this work and all the behaviours are considered under linear elastic conditions. 
2.2 Stress, strain and constitutive relations

The phonon strain $\varepsilon_{i j}$ is defined as in conventional theory of elasticity, however the phason displacement is a function of phonon coordinates only, i.e. $W_{i}\left(x_{i}^{\|}\right)$, and the phason strain $w_{i j}$ isn't symmetric by nature:

$\varepsilon_{i j}=\frac{1}{2}\left(u_{i, j}+u_{j, i}\right), \quad w_{i j}=W_{i, j} \neq W_{j, i}$.

When the atom sequence rearranges going along with phason displacement, it needs energy or force to accomplish it. Hence, the phason traction $h_{i}$ is introduced in complementary space. Accordingly, a 2 nd order phason stress tensor $H_{i j}$ is defined, being related as

$h_{i}=H_{i j} n_{j}$

the first index being attributed to the complementary stress, the second to the physical space where the unit normal $n_{j}$ is defined. Just as phason strain, phason stress thus lacks symmetry, i.e.

$H_{i j} \neq H_{j i}$

The elastic free energy of a $\mathrm{QC}$ is

$$
\begin{aligned}
\Phi & =\Phi_{u u}+\Phi_{w w}+\Phi_{u w} \\
& =\frac{1}{2} C_{i j k l} \varepsilon_{i j} \varepsilon_{k l}+\frac{1}{2} K_{i j k l} w_{i j} w_{k l}+R_{i j k l} \varepsilon_{i j} w_{k l},
\end{aligned}
$$

where $\Phi_{u u}, \Phi_{w w}$ and $\Phi_{u w}$ are pure phonon, pure phason and coupling energies, respectively, and the independent variables are the strains $\varepsilon_{i j}$ and $w_{i j} . C_{i j k l}$ is the phonon elastic stiffness tensor, $K_{i j k l}$ is the stiffness tensor in the phason space and $R_{i j k l}$ denotes the phononphason coupling tensor. Furthermore, the stiffness and coupling tensors can be expressed by means of partial derivatives, i.e.

$$
\begin{aligned}
C_{i j k l}=\frac{\partial^{2} \Phi}{\partial \varepsilon_{i j} \partial \varepsilon_{k l}}, & C_{i j k l}=C_{k l i j}=C_{i j l k}=C_{j i k l}, \\
K_{i j k l}=\frac{\partial^{2} \Phi}{\partial w_{i j} \partial w_{k l}}, & K_{i j k l}=K_{k l i j}, \\
R_{i j k l}=\frac{\partial^{2} \Phi}{\partial \varepsilon_{i j} \partial w_{k l}}, & R_{i j k l}=R_{j i k l} .
\end{aligned}
$$

Eqs. (11) comprise all symmetry conditions of the constitutive tensors. Since the phason strain is asymmetric, the indices of $R_{i j k l}$ and $K_{i j k l}$ are not completely exchangeable, unlike $C_{i j k l}$. In particular, the Maxwell relation is not satisfied, i.e.

$\frac{\partial \sigma_{i j}}{\partial w_{k l}} \neq \frac{\partial H_{k l}}{\partial \varepsilon_{i j}}$
From Eq. (10) the governing linear constitutive equations of quasicrystals are derived as

$\sigma_{i j}=\frac{\partial \Phi}{\partial \varepsilon_{i j}}=C_{i j k l} \varepsilon_{k l}+R_{i j k l} w_{k l}$,

$H_{i j}=\frac{\partial \Phi}{\partial w_{i j}}=R_{k l i j} \varepsilon_{k l}+K_{i j k l} w_{k l}$,

where the stresses can be expressed in Voigt-notation as follows:

$\sigma_{i j}=\left[\sigma_{11}, \sigma_{22}, \sigma_{33}, \sigma_{23}, \sigma_{31}, \sigma_{12}\right]$,

$H_{i j}=\left[H_{11}, H_{22}, H_{33}, H_{23}, H_{31}, H_{12}, H_{32}, H_{13}, H_{21}\right]$.

3 Linear elastic fracture mechanics of quasicrystals

3.1 Stress intensity factors and near tip solutions

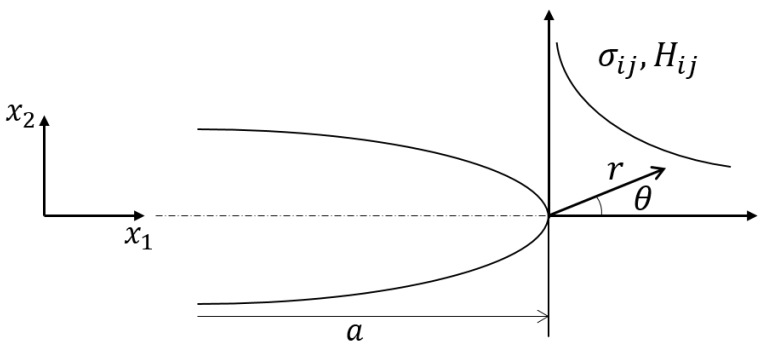

Fig. 2 Polar coordinates $(r, \theta)$ in the vicinity of an opened crack tip and the stresses $\sigma_{i j}, H_{i j}$ on the ligament; $a$ is the half length of an interior crack.

Within the framework of linear elasticity of QCs, the phason as well as the phonon stresses exhibit singularities at crack tips [37], see Fig. 2. By approaching the crack tip, the stress intensity factors for the phonon field $\left[K_{I I}^{\|}, K_{I}^{\|}, K_{I I I}^{\|}\right]$and the phason field $\left[K_{I I}^{\perp}, K_{I}^{\perp}, K_{I I I}^{\perp}\right]$ are defined as follows

$$
\begin{aligned}
& K_{i}^{\|}=\left[K_{I I}^{\|}, K_{I}^{\|}, K_{I I I}^{\|}\right]^{T}=\lim _{r \rightarrow 0} \sqrt{2 \pi r} \sigma_{i 2}(r, \theta=0), \\
& K_{i}^{\perp}=\left[K_{I I}^{\perp}, K_{I}^{\perp}, K_{I I I}^{\perp}\right]^{T}=\lim _{r \rightarrow 0} \sqrt{2 \pi r} H_{i 2}(r, \theta=0) .
\end{aligned}
$$

For the sake of compactness, the intensity factors of phonon and phason fields can be merged into one single vector

$$
\begin{aligned}
K_{P} & =\left[K_{I I}^{\|}, K_{I}^{\|}, K_{I I I}^{\|}, K_{I I}^{\perp}, K_{I}^{\perp}, K_{I I I}^{\perp}\right]^{T} \\
& =\left\{\begin{array}{cc}
K_{i}^{\|} & \text {if } 1 \leq P \leq 3 \\
K_{i}^{\perp} & \text { if } 4 \leq P \leq 6
\end{array} .\right.
\end{aligned}
$$

The same nomenclature holds for the stress, the displacement and the strain, where uppercase indices run from 1 to 6 and $1,2,3(4,5,6)$ refers to the phonon (phason) field. 
Gao et al. 38] have derived the stress functions for a Griffith crack, i.e. a crack in an infinite plate under stress loading $\sigma_{J 2}^{\infty}$ :

$$
\begin{aligned}
\sigma_{I 1} & =\left[\sigma_{11}, \sigma_{21}, \sigma_{31}, H_{11}, H_{21}, H_{31}\right]^{T} \\
& =\sum_{N=1}^{6} \Re\left\{B_{I N}\left(p_{N}-\frac{z_{N} p_{N}}{\sqrt{z_{N}^{2}-a^{2}}}\right) B_{N J}^{-1}\right\} \sigma_{J 2}^{\infty}, \\
\sigma_{I 2} & =\left[\sigma_{12}, \sigma_{22}, \sigma_{32}, H_{12}, H_{22}, H_{32}\right]^{T} \\
& =\sum_{N=1}^{6} \Re\left\{B_{I N} \frac{z_{N}}{\sqrt{z_{N}^{2}-a^{2}}} B_{N J}^{-1}\right\} \sigma_{J 2}^{\infty}, \\
u_{I} & =\left[u_{1}, u_{2}, u_{3}, W_{1}, W_{2}, W_{3}\right]^{T} \\
& =\sum_{N=1}^{6} \Re\left\{A_{I N}\left(\sqrt{z_{N}^{2}-a^{2}}-z_{N}\right) B_{N J}^{-1}\right\} \sigma_{J 2}^{\infty},
\end{aligned}
$$

where $z_{N}=a+r\left(\cos \theta+p_{N} \sin \theta\right)$ is a conformal mapping function and the matrices $A_{I N}$ and $B_{I N}$ as well as the eigenvalues $p_{N}$ are characterized by material constants (see appendix). The real part of a complex quantity is denoted as $\mathfrak{R}\{\ldots\}$. The stress intensity factors of the Griffith crack problem are obtained as $K_{P}=\sigma_{P 2}^{\infty} \sqrt{\pi a}$, inserting Eqs. (17) into Eqs. (15). Further, the general near-tip solutions are derived from Eqs. (17) taking the limit $r \rightarrow 0$ :

$$
\begin{aligned}
\sigma_{I 1} & =-\frac{1}{\sqrt{2 \pi r}} \Re\left\{B_{I N} P_{N M}^{\mathrm{S} 1} B_{M J}^{-1}\right\} K_{J}, \\
\sigma_{I 2} & =\frac{1}{\sqrt{2 \pi r}} \Re\left\{B_{I N} P_{N M}^{\mathrm{S} 2} B_{M J}^{-1}\right\} K_{J}, \\
u_{I} & =\sqrt{\frac{2 r}{\pi}} \Re\left\{A_{I N} P_{N M}^{\mathrm{u}} B_{M J}^{-1}\right\} K_{J},
\end{aligned}
$$

with the diagonal matrices $P_{N M}$ according to the appendix.

\subsection{Energy release rate and crack closure integral}

According to the general definition of the energy release rate, $G$ is the total potential energy per unit surface reduced by crack propagation. The energy release rate can be expressed as follows

$G=\lim _{\Delta A \rightarrow 0} \frac{-\Delta \Pi}{\Delta A}=-\frac{d \Pi}{d A}$,

where $\Delta \Pi=-\Delta W^{s}$ is the reduced total potential energy of the system being equal to the negative work which is required for crack closure, in case of a reversal of the quasi-static process of crack growth [39]. It can thus be considered as the work which is needed to close the crack along a segment $\Delta A$. Hence, in QCs not only the phonon but also the phason part are involved in this work. The local work at a point $r$ on the dashed crack faces $(\theta=0)$, see Fig. 3, which is required for crack closure, is calculated as

$$
\begin{gathered}
d W^{s}(r, 0)=\int_{u_{i}^{+}}^{0} F_{i}^{\|+} d x_{i}^{\|}+\int_{W_{i}^{+}}^{0} F_{i}^{\perp+} d x_{i}^{\perp} \\
\quad+\int_{i}^{0} F_{i}^{\|-} d x_{i}^{\|}+\int_{W_{i}^{-}}^{0} F_{i}^{\perp-} d x_{i}^{\perp} \\
=-\frac{\bar{F}_{i}^{\|+} u_{i}^{+}}{2}-\frac{\bar{F}_{i}^{\perp+} W_{i}^{+}}{2} \\
\quad-\frac{\bar{F}_{i}^{\|-} u_{i}^{-}}{2}-\frac{\bar{F}_{i}^{\perp-} W_{i}^{-}}{2},
\end{gathered}
$$

where the phonon and phason forces $F_{i}^{\|}$and $F_{i}^{\perp}$ effectuate respective displacements on positive and negative crack faces $x_{i}^{\|} \in\left[u_{i}^{ \pm}, 0\right]$ and $x_{i}^{\perp} \in\left[W_{i}^{ \pm}, 0\right]$. When the crack is completely closed, the forces $\bar{F}_{i}^{\|}$and $\bar{F}_{i}^{\perp}$ are those on the created ligament at position $r$. The outward unit normal vectors of the crack faces being $n_{i}=(0, \mp 1)^{T}$, the forces are related to the stresses as $\bar{F}_{i}^{\|}=\bar{\sigma}_{2 i} n_{2} d A=$ $\mp \bar{\sigma}_{2 i} d A$ and $\bar{F}_{i}^{\perp}=\mp \bar{H}_{i 2} d A$, where the upper sign (-) stands for the positive and the lower sign $(+)$ for the negative crack surface.

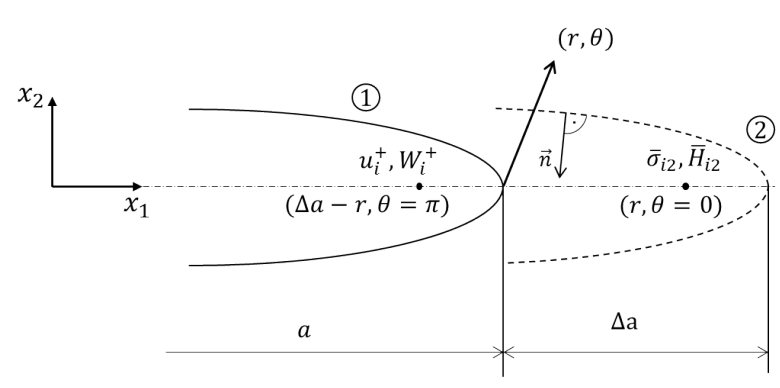

Fig. 3 Derivation of the crack closure integral: the solid line represents the current crack tip (1), the dashed line the state after crack growth (2).

From Eq. (20) the local crack closure work is thus obtained as

$$
\begin{aligned}
d W^{s} & =\frac{1}{2} \bar{\sigma}_{2 i}\left(u_{i}^{+}-u_{i}^{-}\right) d A+\frac{1}{2} \bar{H}_{i 2}\left(W_{i}^{+}-W_{i}^{-}\right) d A \\
& =\frac{1}{2} \bar{\sigma}_{I 2}\left(u_{I}^{+}-u_{I}^{-}\right) d A .
\end{aligned}
$$

Assuming that the displacements for both fields are symmetric with respect to the positive and negative crack surfaces, i.e. $u_{I}^{+}=-u_{I}^{-}$, the crack closure work for a finite crack surface $\Delta A$ is determined by integration:

$$
\Delta W^{s}=-\Delta \Pi=\int_{\Delta A} \bar{\sigma}_{I 2} u_{I}^{+} d A=B \int_{\Delta a} \bar{\sigma}_{I 2} u_{I}^{+} d x_{1} .
$$


Plane crack problems imply $\Delta A=B \Delta a$ and $d A=B d x_{1}=$ $B d r$ with $B$ as constant thickness. Refering to Fig. 3 , where the crack opening displacements are taken from the crack faces at $(\Delta a-r, \theta=\pi)$ and the tractions are taken from the ligament at $(r, \theta=0)$ [39], where the bars over the variables can be omitted, the energy release rate for a plane problem is introduced inserting Eq. (22) into Eq. (19):

$$
\begin{gathered}
G=\lim _{\Delta a \rightarrow 0} \frac{1}{\Delta a} \int_{0}^{\Delta a}\left\{\sigma_{i 2}(r, 0) u_{i}^{+}(\Delta a-r, \pi)\right. \\
\left.+H_{i 2}(r, 0) W_{i}^{+}(\Delta a-r, \pi)\right\} d r .
\end{gathered}
$$

The limit $\Delta a \rightarrow 0$ is essential taking displacements and stresses from two different crack lengths, i.e. $a$ and $a+\Delta a$, see Fig. 3. Consequently, crack tip near fields according to Eqs. (18) may be applied to Eq. (23) for a crack length $a$.

Following ideas from conventional fracture mechanics [40], a second energy term $H$ is defined by formally replacing $\sigma_{i 2}$ and $H_{i 2}$ by $\sigma_{i 1}$ and $H_{i 1}$ :

$$
\begin{gathered}
H=\lim _{\Delta a \rightarrow 0} \frac{1}{\Delta a} \int_{0}^{\Delta a}\left\{\sigma_{i 1}(r, 0) u_{i}^{+}(\Delta a-r, \pi)\right. \\
\left.+H_{i 1}(r, 0) W_{i}^{+}(\Delta a-r, \pi)\right\} d r .
\end{gathered}
$$

Substituting Eqs. (18) into Eqs. (23) and (24), the relations between stress intensity factors and the two energy rates $G$ and $H$ are derived. In compact notation they read

$$
\begin{aligned}
G & =\lim _{\Delta a \rightarrow 0} \frac{1}{\Delta a} \int_{0}^{\Delta a} \sqrt{\frac{\Delta a-r}{\pi^{2} r}} d r K_{P} Y_{P Q} K_{Q}=\frac{1}{2} K_{P} Y_{P Q} K_{Q}, \\
H & =\lim _{\Delta a \rightarrow 0} \frac{1}{\Delta a} \int_{0}^{\Delta a}-\sqrt{\frac{\Delta a-r}{\pi^{2} r}} d r K_{P} \Re\left\{B_{P N}^{-1} P_{N M}^{\mathrm{S} 1}(\theta=0) B_{M J}\right\} Y_{J Q} K_{Q} \\
& =-\frac{1}{2} K_{P} \Re\left\{B_{P N}^{-1} P_{N M}^{\mathrm{S} 1}(\theta=0) B_{M J}\right\} Y_{J Q} K_{Q},
\end{aligned}
$$

where

$$
Y_{P Q}=\mathfrak{R}\left\{i A_{P N} B_{N Q}^{-1}\right\}
$$

is the generalized Irwin matrix for QCs, and the crack opening displacement can be expressed as

$u_{P}=\left[u_{i}, W_{i}\right]^{T}= \pm \sqrt{\frac{2 r}{\pi}} Y_{P Q} K_{Q}$.

Whereas a unique physical meaning is attributed to the energy release rate $G$, an interpretation of $H$ with respect to crack closure is not known. It is rather an auxiliary quantity, which may be useful e.g. in numerical fracture analysis [41], in particular in connection with the J-integral.

\subsection{Configurational force and J-integral}

In the following, a straightforward but rigorous derivation of the J-integral is depicted, starting from the first law of thermodynamics

$d U=d W^{\mathrm{rev}}+d Q+d \Psi$,

where the kinetic energy is neglected, $U$ is the internal energy, $d Q$ the heat flux and the work of external forces is separated into a reversible part $d W^{\text {rev }}$ and the dissipation $d \Psi$. Inserting $d \hat{W}^{\text {rev }}=\sigma_{M N} d \varepsilon_{M N}, d \hat{Q}=T d \hat{S}^{a}$ and $d \hat{\Psi}=T d \hat{S}^{i}$, where $T$ is the absolute temperature and $\hat{S}^{a}$ and $\hat{S}^{i}$ are exchange and irreversible specific entropies, respectively, Eq. (27) yields the Gibbs fundamental equation for the specific internal energy

$d \hat{U}=\sigma_{i j} d \varepsilon_{i j}+H_{i j} d W_{i j}+T d \hat{S}$.

Neglecting heat flux, i.e. $d \hat{S}^{a}=0$, the specific dissipative work is obtained as

$d \hat{\Psi}=d \Phi-\sigma_{i j} d \varepsilon_{i j}-H_{i j} d W_{i j}$,

where the more general $\hat{U}$ has been replaced by the specific potential of QC according to Eq. (10).

A QC continuum is considered first including a point defect with a virtual displacement $\delta z_{k}$ in a control volume $V$. According to Eq. (29), the associated dissipative work is given as

$J_{k} \delta z_{k}=\int_{V}\left(\delta \Phi-\sigma_{i j} \delta \varepsilon_{i j}-H_{i j} \delta W_{i j}\right) d V$,

where $J_{k}$ is a generalized or configurational force acting at the defect. Considering the balance equations $\sigma_{I j, j}=0$, where volume forces and mass related inertia are neglected, the phonon and phason virtual works are transformed as

$\sigma_{i j} \delta \varepsilon_{i j}=\left(\sigma_{i j} u_{i, k}\right)_{, j} \delta z_{k}$,

$H_{i j} \delta w_{i j}=\left(H_{i j} W_{i, k}\right),{ }_{j} \delta z_{k}$,

where obviously the virtual displacement $\delta z_{k}$ is in the phonon field. With

$\delta \Phi=\frac{d \Phi}{d x_{k}} \delta z_{k}=\left(\Phi \delta_{k j}\right)_{, j} \delta z_{k}$,

the configurational force of QCs is obtained from Eq. (30) by eliminating $\delta z_{k}$ :

$J_{k}=\int_{V}\left(\Phi \delta_{k j}-\sigma_{i j} u_{i, k}-H_{i j} W_{i, k}\right), j d V$.

The terms in the round brackets of Eq. (32) constitute the generalized energy-momentum or Eshelby tensor [42] for quasicrystals,

$Q_{k j}=\Phi \delta_{k j}-\sigma_{i j} u_{i, k}-H_{i j} W_{i, k}$. 
The configurational force is transformed into a contour integral by using the divergence theorem introducing the outward unit normal $n_{j}$ :

$J_{k}=\oint_{\Gamma} Q_{k j} n_{j} d \Gamma$.

The integral is path independent as long as the contour $\Gamma$ encloses the defect and is zero otherwise.

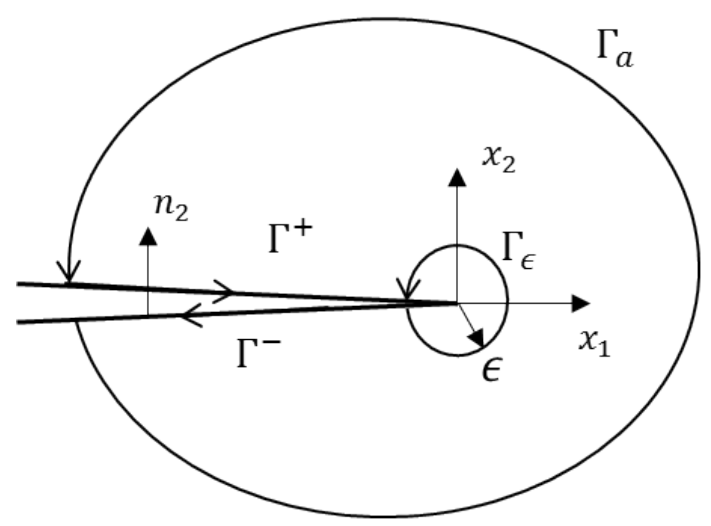

Fig. 4 Contours for the J-integral; arrows indicate the orientations of the integration paths.

Applying Eqs. (32) or (34) to a crack problem, the configurational force $J_{k}$ is denoted as J-integral. According to Fig. 1, a closed contour can be constructed including both crack surfaces $\Gamma^{+}, \Gamma^{-}$, however excluding the crack tip. Thus, the J-integral vanishes:

$J_{k}=\oint_{\Gamma} Q_{k j} n_{j} d \Gamma=\oint_{\Gamma_{a}+\Gamma^{+}-\Gamma_{\varepsilon}+\Gamma^{-}} Q_{k j} n_{j} d \Gamma=0$.

On the other hand, the J-integral can be calculated just from the inner contour $\Gamma_{\varepsilon}$, as long as it is sufficiently small:

$$
\begin{aligned}
J_{k}= & \lim _{\varepsilon \rightarrow 0} \int_{\Gamma_{\varepsilon}} Q_{k j} n_{j} d \Gamma \\
= & \lim _{\varepsilon \rightarrow 0} \int_{\Gamma_{a}} Q_{k j} n_{j} d \Gamma \\
& \quad+\lim _{\varepsilon \rightarrow 0} \int_{\Gamma^{+}+\Gamma^{-}}\left(\Phi n_{k}-t_{i} u_{i, k}-h_{i} W_{i, k}\right) d \Gamma .
\end{aligned}
$$

The second equality in Eq. (36) emanates from Eq. (35). An integration along the crack faces is always required in connection with a finite contour $\Gamma_{a}$, if there are tractions $t_{i}, h_{i}$ on the crack faces. In a traction-free case just the first term with the potential $\Phi$ remains, guaranteeing path-independence of the J-integral. Just if the crack, in addition, is straight and only $J_{1}$ or $J_{2}$ are of interest, a crack surface integration does not apply if the coordinate system is appropriately chosen.

From Eq. (36) the two coordinates of the plane Jintegral are obtained as

$$
\begin{aligned}
& J_{1}=\lim _{\varepsilon \rightarrow 0} \int_{\Gamma_{\varepsilon}}\left(\Phi \delta_{1 j}-\sigma_{i j} u_{i, 1}-H_{i j} W_{i, 1}\right) n_{j} d \Gamma, \\
& J_{2}=\lim _{\varepsilon \rightarrow 0} \int_{\Gamma_{\varepsilon}}\left(\Phi \delta_{2 j}-\sigma_{i j} u_{i, 2}-H_{i j} W_{i, 2}\right) n_{j} d \Gamma .
\end{aligned}
$$

The energy release rate and the $\boldsymbol{J}_{k}$-vector are generally related by the unit vector of crack growth direction $z_{k}$ :

$G=J_{k} z_{k}$.

If, in particular, the local orthogonal coordinate system $\left(x_{1}, x_{2}\right)$, see Fig. 4 , is chosen in such a way that the crack surfaces are parallel to the $x_{1}$-axis and furthermore the crack propagates into the $x_{1}$-direction, thus $z_{2}=0$, the contour integrals according to Eq. (37) correspond to the energy release rate $G$ and $H$ as

$G=J_{1}, \quad H=-J_{2}$,

which is confirmed by integration according to Eqs. (37) and (23), (24) or in connection with Eq. (25). These relationships are consistent with those of classical elastic materials [40].

\subsection{Crack deflection criteria at mixed-mode loading}

If only the simple mode-I case due to symmetrical loading and geometry is considered, in classical fracture mechanics the crack grows straightforward along its ligament. Under mixed-mode loading, in a sense of unsymmetrical loading, a few criteria have been developed to predict the crack growth deflection. Two of them are applied here and implemented for QC. The first one is the criterion of maximum circumferential stress [43]. There the crack grows into the one direction $\bar{\theta}$ where the circumferential stress $\sigma_{\theta \theta}$ reaches its maximum value, see Fig. 5 .

This criterion can be easily applied if the crack tip stress is given as a function $\sigma_{\theta \theta}=f(\theta)$, i.e.

$\left.\frac{\partial \sigma_{\theta \theta}}{\partial \theta}\right|_{\theta=\bar{\theta}}=0,\left.\quad \frac{\partial^{2} \sigma_{\theta \theta}}{\partial \theta^{2}}\right|_{\theta=\bar{\theta}}<0$.

Since $r$ may be infinitely small, asymptotic crack tip near field stresses are employed with Eq. (40), being related to the stress intensity factors according to Eq. (18). While for crystals the definition of stress is unique, QCs are subject to phonon and phason stress. A crack deflection or fracture criterion could exploit either the one or the other stress or introduce an equivalent stress 


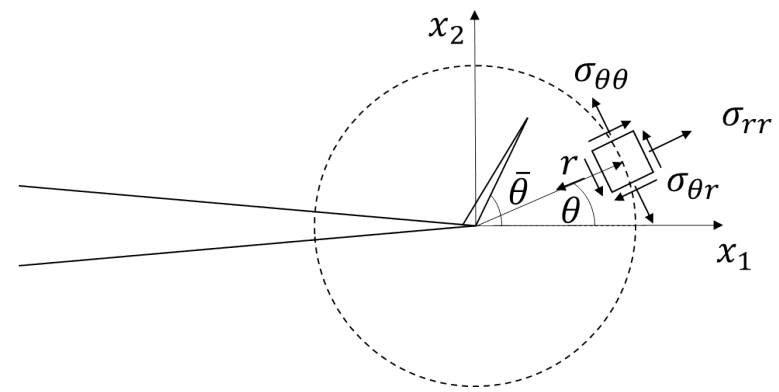

Fig. 5 Stress state in polar coordinates around a crack tip to illustrate the criterion of maximum circumferential stress.

criterion of any kind whatsoever. In the present study, fracture is supposed to occur just in physical space, thus phonon stress is relevant in Eq. (40), of course depending on the phason stress due to coupling, see Eq. (13).

The second hypothesis is the J-integral criterion. It postulates that the crack extends along the direction of the configurational force, see Fig. 6. According to Eq. (38) the scalar product $J_{k} z_{k}$ is maximal if $z_{k}$ and $J_{k}$ are linearly dependent, thus leading to a maximum of the energy release rate. The deflection angle is accordingly determined as

$\bar{\theta}=\arctan \left(J_{2} / J_{1}\right)$.

In the local crack tip coordinate system, again $x_{1}$ is tangential to the crack faces and $x_{2}$ is perpendicular. The coordinates of the J-integral can be computed either from the path-independent integral Eq. (36) or alternatively be derived from the crack closure integral, see Eqs. (39) and (23), (24). Accounting for Eqs. (25), the $J_{k}$-vector is related to the stress intensity factors as

$J_{1}=\frac{1}{2} K_{P} Y_{P Q} K_{Q}$,

$J_{2}=\frac{1}{2} K_{P} \Re\left\{B_{P N}^{-1} P_{N M}^{\mathrm{S} 1}(\theta=0) B_{M J}\right\} Y_{J Q} K_{Q}$.

Deriving Eq. (42) directly from Eq. (37) inserting the asymptotic near tip fields is not possible since the integration does not provide a closed-form solution.

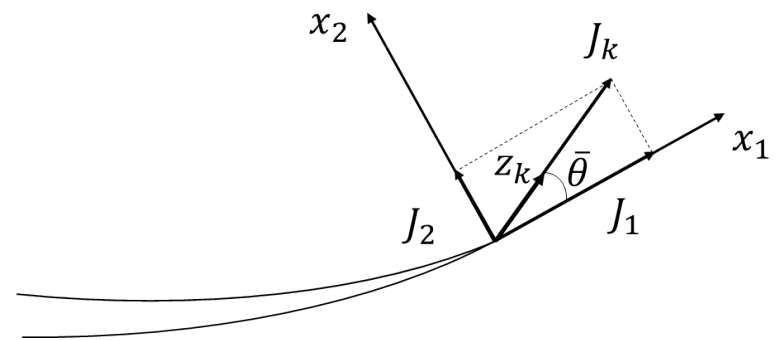

Fig. 6 J-integral criterion for the crack deflection angle $\bar{\theta}$.
4 Results of crack deflection in QCs

In this section the selected fracture and deflection criteria are implemented for $1 \mathrm{D}$ QC with an initial horizontal cleavage, see Fig. 5. Since there is only one quasiperiodic direction in a $1 \mathrm{D}$ QC, different configurations are possible for plane problems. The quasiperiodic axis (QA) can be perpendicular to the plane, thus leading to crystalline behaviour. The QA being in the plane of interest arbitrary orientations are possible with respect to a crack. Here, two configurations are selected, i.e. the QA is parallel or perpendicular to the crack faces. The material constants and the matrices related to the closed-form solution for the two states are given in the appendix.

Applying the criterion of maximum circumferential stress, Eq. (40), the near tip solution, Eq. (18), has to be transformed into polar coordinates following the transformation

$$
\begin{gathered}
\left(\begin{array}{ccc}
\sigma_{r r} & \sigma_{r \theta} & 0 \\
\sigma_{\theta r} & \sigma_{\theta \theta} & 0 \\
0 & 0 & 0
\end{array}\right)= \\
\left(\begin{array}{ccc}
\cos (\theta) & \sin (\theta) & 0 \\
-\sin (\theta) & \cos (\theta) & 0 \\
0 & 0 & 1
\end{array}\right)\left(\begin{array}{ccc}
\sigma_{11} & \sigma_{12} & 0 \\
\sigma_{21} & \sigma_{22} & 0 \\
0 & 0 & 0
\end{array}\right)\left(\begin{array}{ccc}
\cos (\theta) & -\sin (\theta) & 0 \\
\sin (\theta) & \cos (\theta) & 0 \\
0 & 0 & 1
\end{array}\right) .
\end{gathered}
$$

The circumferential stress is thus given as

$$
\begin{aligned}
\sigma_{\theta \theta}= & \cos (\theta)\left(\sigma_{22} \cos (\theta)-\sigma_{12} \sin (\theta)\right) \\
& -\sin (\theta)\left(\sigma_{21} \cos (\theta)-\sigma_{11} \sin (\theta)\right),
\end{aligned}
$$

where the stress components related to $x_{3}$ are zero due to plane stress conditions. In this process, the phason stress does not have to be transformed into polar coordinates, since in the criterion of maximum circumferential stress only the phonon stress $\sigma_{\theta \theta}$ is assumed to have an influence on crack deflection. The deflected angle $\bar{\theta}$ is determined by inserting Eq. (44) into Eq. (40).

Considering the J-integral criterion Eq. (41), $J_{1}$ and $J_{2}$ are required. In order to keep the formulation most general and to abstain from specific boundary value problems, Eq. (42) is taken into account expressing the loading in terms of stress intensity factors. Based on the material constants given in the appendix, the coordinates of $J_{k}$ are determined as

$$
\begin{aligned}
J_{1}= & \left(5.1066 K_{I}^{\| 2}+5.1897 K_{I I}^{\| 2}-0.08824 K_{I I}^{\|} K^{\perp}\right. \\
& \left.+9.2413 K^{\perp 2}\right) \times 10^{-12}, \\
J_{2}=- & 1.0213 \times 10^{-11} K_{I}^{\|} K_{I I}^{\|}-3.1194 \times 10^{-25} K_{I}^{\|} K^{\perp},
\end{aligned}
$$


$(45)$

for the QA being parallel to the crack faces and

$$
\begin{aligned}
J_{1}=\left(5.1897 K_{I}^{\| 2}+5.1066 K_{I I}^{\| 2}-0.08824 K_{I}^{\|} K^{\perp}\right. & \\
& \left.+9.2413 K^{\perp 2}\right) \times 10^{-12}, \\
J_{2}=- & 1.0379 \times 10^{-11} K_{I}^{\|} K_{I I}^{\|}-2.2198 \times 10^{-14} K_{I I}^{\|} K^{\perp},
\end{aligned}
$$

for the QA being perpendicular. The phason stress intensity factor $K^{\perp}$ is written without subscript index since it is a unique quantity in $1 \mathrm{D}$ QC. Eqs. (45) and (46) reveal that the coefficients related to mixed terms including both phonon and phason stress intensity factors are smaller than those of pure phonon or phason terms. The second term in $J_{2}$ in Eq. (45) can be completely neglected, while according to Eq. (46) $K^{\perp}$ might bring some contribution to $J_{2}$ for a perpendicular QA. Furthermore, the impact of $K^{\perp}$ on $J_{1}$ has about the same significance as the one of $K_{I I}^{\|}$or $K_{I}^{\|}$. In conventional fracture mechanics, a $K_{I I}$ being even small compared to $K_{I}$, influences crack growth significantly, leading to a crack deflection from the initial ligament [44]. This observation gives rise to the presumption that $K^{\perp}$ will show a similar effect on crack propagation.

In literature only material constants of $2 \mathrm{D}$ and $3 \mathrm{D}$ QCs are given, however there isn't any experimental result for 1D QC. Therefore, parameters of the 2D QC $\mathrm{Al}-\mathrm{Ni}$-Co have been adopted here. The $R_{3}$ missing in $2 \mathrm{D}$ $\mathrm{QC}$ is furthermore assumed to be in the same order of magnitude as $R_{2}$. The compressed notation of $R_{i j k l}$ is depicted in Eq. (47). Concerning the coupling constants, those being available represent just a few materials and predominantly evolve from ab initio or molecular dynamics simulations [45], rather than being experimental findings. Thus, they have to be considered not to be quite reliable, whereupon a set of enlarged constants is additionally used to investigate the influence of the phason field.

The calculated crack deflections $\bar{\theta}$ are now compared, where the quasiperiodic axis is parallel, see Tab. 1, or perpendicular to the cleavage, see Tab. 2. Each deflection criterion has two columns. The first shows the results based on coupling constants according to the appendix and in the second column, the coupling constants are 50 times enlarged. Stress intensity factors not explicitly given in the loading column are zero, so the first two rows are single mode and the rest are mixedmode loadings. The J-integral criterion, being known to be valid only if $K_{I}$ is dominating $K_{I I}[39]$, hasn't been applied for $K_{I}^{\|}=0 . K^{\perp}$ alone is not applied, since phason loading probably isn't possible, the phason stress intensity factor rather being induced by the coupling

\begin{tabular}{|c|c|c|c|c|}
\hline \multirow{3}{*}{ loading } & \multicolumn{4}{|c|}{ crack deflection $\bar{\theta}$} \\
\hline & \multicolumn{2}{|c|}{ J-integral criterion } & \multicolumn{2}{|c|}{ circumferential stress c. } \\
\hline & $\mathrm{R}$ & $50 \times \mathrm{R}$ & $\mathrm{R}$ & $50 \times \mathrm{R}$ \\
\hline$K_{I}^{\|}$ & $0^{\circ}$ & $0^{\circ}$ & $0^{\circ}$ & $0^{\circ}$ \\
\hline$K_{I I}^{\|}$ & - & - & $-71.03^{\circ}$ & $-72.13^{\circ}$ \\
\hline$K_{I I}^{\|}=K^{\perp}$ & - & - & $-71^{\circ}$ & $-69.82^{\circ}$ \\
\hline$K_{I}^{\|}=K^{\perp}$ & $\approx 0^{\circ}$ & $\approx 0^{\circ}$ & $\approx 0^{\circ}$ & $\approx 0^{\circ}$ \\
\hline$K_{I I}^{\|}=1 \% K_{I}^{\|}$ & $-1.15^{\circ}$ & $-1.15^{\circ}$ & $-1.13^{\circ}$ & $-1.19^{\circ}$ \\
\hline $\begin{aligned} K_{I I}^{\|} & =1 \% K_{I}^{\|} \\
K^{\perp} & =1 \% K_{I}^{\|}\end{aligned}$ & $-1.15^{\circ}$ & $-1.15^{\circ}$ & $-1.13^{\circ}$ & $-1.18^{\circ}$ \\
\hline $\begin{array}{c}K_{I I}^{\|}=1 \% K_{I}^{\|} \\
K^{\perp}=10 \% K_{I}^{\|}\end{array}$ & $-1.13^{\circ}$ & $-1.12^{\circ}$ & $-1.13^{\circ}$ & $-1.18^{\circ}$ \\
\hline $\begin{array}{c}K_{I I}^{\|}=1 \% K_{I}^{\|} \\
K^{\perp}=50 \% K_{I}^{\|}\end{array}$ & $-0.79^{\circ}$ & $-0.72^{\circ}$ & $-1.13^{\circ}$ & $-1.18^{\circ}$ \\
\hline $\begin{array}{c}K_{I I}^{\|}=1 \% K_{I}^{\|} \\
K^{\perp}=100 \% K_{I}^{\|}\end{array}$ & $-0.41^{\circ}$ & $-0.34^{\circ}$ & $-1.13^{\circ}$ & $-1.17^{\circ}$ \\
\hline
\end{tabular}

Table 1 Comparison of the crack deflection under different mixed-mode loadings where the QA is parallel to the cleavage.

Table 2 Comparison of the crack deflection under different

\begin{tabular}{|c|c|c|c|c|}
\hline \multirow{3}{*}{ loading } & \multicolumn{4}{|c|}{ crack deflection $\bar{\theta}$} \\
\hline & \multicolumn{2}{|c|}{ J-integral criterion } & \multicolumn{2}{|c|}{ circumferential stress c. } \\
\hline & $\mathrm{R}$ & $50 \times \mathrm{R}$ & $\mathrm{R}$ & $50 \times \mathrm{R}$ \\
\hline$K_{I}^{\|}$ & $0^{\circ}$ & $0^{\circ}$ & $0^{\circ}$ & $0^{\circ}$ \\
\hline$K_{I I}^{\|}$ & - & - & $-71.32^{\circ}$ & $-70.95^{\circ}$ \\
\hline$K_{I I}^{\|}=K^{\perp}$ & - & - & $-71.33^{\circ}$ & $-71.54^{\circ}$ \\
\hline$K_{I}^{\|}=K^{\perp}$ & $0^{\circ}$ & $0^{\circ}$ & $0^{\circ}$ & $0^{\circ}$ \\
\hline$K_{I I}^{\|}=1 \% K_{I}^{\|}$ & $-1.15^{\circ}$ & $-0.96^{\circ}$ & $-1.16^{\circ}$ & $-1.07^{\circ}$ \\
\hline $\begin{aligned} K_{I I}^{\|} & =1 \% K_{I}^{\|} \\
K^{\perp} & =1 \% K_{I}^{\|}\end{aligned}$ & $-1.15^{\circ}$ & $-0.97^{\circ}$ & $-1.16^{\circ}$ & $-1.07^{\circ}$ \\
\hline $\begin{array}{c}K_{I I}^{\|}=1 \% K_{I}^{\|} \\
K^{\perp}=10 \% K_{I}^{\|}\end{array}$ & $-1.13^{\circ}$ & $-1.07^{\circ}$ & $-1.16^{\circ}$ & $-1.09^{\circ}$ \\
\hline $\begin{array}{c}K_{I I}^{\|}=1 \% K_{I}^{\|} \\
K^{\perp}=50 \% K_{I}^{\|}\end{array}$ & $-0.80^{\circ}$ & $-1.02^{\circ}$ & $-1.17^{\circ}$ & $-1.15^{\circ}$ \\
\hline $\begin{array}{c}K_{I I}^{\|}=1 \% K_{I}^{\|} \\
K^{\perp}=100 \% K_{I}^{\|}\end{array}$ & $-0.42^{\circ}$ & $-0.53^{\circ}$ & $-1.17^{\circ}$ & $-1.24^{\circ}$ \\
\hline
\end{tabular}
mixed-mode loadings where the QA is perpendicular to the cleavage.

effect under phonon loading. In Tabs. 1 and 2 only ratios of stress intensity factors are given, since absolute values are not relevant for the deflection angle $\bar{\theta}$, which is obvious looking at Eqs. (45) or (46).

For a pure phonon mode-I loading there is no crack deflection, no matter which magnitude of the coupling constant R is chosen. Even a phason loading as large as $K_{I}$ doesn't show any significant impact on the deflection angle. Focusing first on the J-integral criterion, Eqs. (45) and (46) illustrate that $J_{2}$ is only non-vanishing, if there is a mode-II loading. A finite $J_{2}$, however, is indispensable for a crack deviating from a straight propaga- 
tion. Most of the examples in Tabs. 1 and 2 thus exhibit a phonon mixed mode loading where $K_{I I}$, however, is minor compared to $K_{I}$. The small value being just $1 \%$ of $K_{I}$ was chosen in an order of magnitude which is typical along curved crack paths in inhomogeneous stress fields under constant external loading as observed e.g. near holes or inclusions [44]. The deflection angle predicted by the J-integral for pure phonon loading in this case is $-1.15^{\circ}$. It is remarkable that a larger phononphason coupling of $50 \times \mathrm{R}$, in the case of the QA being perpendicular to the initial crack, leads to a smaller angel of $-0.96^{\circ}$. In Eq. (46) the influence of the coupling constant is not obvious, whereas Eq. (42) shows material dependent matrices in the relation of the J-integral and the stress intensity factors. In Eqs. (45) and (46) the material dependence and thus the influence of the coupling coefficient is found in the algebraic factors. In Tabs. 1 and 2 the last four rows show results for increasing phason loads superimposing a phonon modeI/II loading, finally leading to a considerable reduction of the deflection angle. After all, the influence of $K^{\perp}$ on crack deflection is smaller than the one of $K_{I I}$ since the related algebraic factors for $J_{2}$ in Eqs. (45) and (46) are much smaller and for the perpendicular QA $K^{\perp}$ is multiplied by $K_{I I}$ instead of by the larger $K_{I}$.

Concerning the maximum circumferential stress criterion, a crack deflection under pure mode-II loading is investigated. The predicted angles are slightly larger than the value for classical isotropic elasticity $(\bar{\theta}=$ $\left.-70.5^{\circ}\right)$. For the decoupled case of anisotropic elasticity $(\mathrm{R}=0), \bar{\theta}=-71.03^{\circ}$ and $\bar{\theta}=-71.32^{\circ}$ are calculated for the QA parallel and perpendicular to the crack. If a phason load is superimposed, a remarkable change of angles is not observed. For the mode-I/II loading cases the magnitudes of the angles are very close to those predicted by the J-integral criterion. In contrast to the latter criterion, a phason loading $K^{\perp}$ doesn't have a considerable influence on the deflection angle, unless larger coupling coefficients $\mathrm{R}$ are employed. Comparing both criteria, the energetic J-integral criterion fully takes into account the energy stored in the phason field and postulates the minimization of the total potential energy of the system, thus following a general law of nature. The maximum circumferential stress criterion as formulated here, on the other hand, attributes the fracture process essentially to the phonon field.

\section{Conclusions and outlook}

The configurational force and the J-integral for QC are derived from the first law of thermodynamics for dissipative processes. The crack closure integral of linear elastic fracture mechanics is generalized to provide a relation between the J-integral vector and the stress intensity factors. Two crack deflection criteria are introduced, i.e. an energy-related based on the J-integral and a stress based one. The latter accounts for the maximum circumferential phonon stress. Due to the phason coupling, a mixed-mode crack tip loading is intrinsic to fracture of QC. The influence of phason loading in terms of the phason stress intensity factor on crack deflection is in the focus of the investigations. Two different orientations of the quasicrystalline axis (QA) of a 1D QC are assumed, one parallel, the other perpendicular to the crack. Concerning the phonon-phason coupling coefficients, which are barely available from literature, two different values are employed differing by a factor of 50 .

One outcome of the investigations is that phason fields only have an impact on the crack deflection, if a mode-II loading is imposed. For pure mode-II, the phonon-phason coupling slightly influences the deflection angle, not exceeding a deviation of $2-3 \%$. The most interesting case, however, is a mode-I/II loading, with $K_{I I}$ being much smaller than $K_{I}$, being characteristic for many crack growth problems. Here, the J-integral criterion predicts a considerable impact on the crack deflection if the coupling coefficients or the phason stress intensity factor are sufficiently large. If the phason stress intensity factor of a predominantly mode-Idriven crack is of the same order of magnitude as $K_{I I}$, the influence of the phason field on the crack path is smaller than expected, unless the coupling coefficient is much larger than the very few values nowadays being available in literature for $2 \mathrm{D}$ or $3 \mathrm{D} \mathrm{QC}$.

Generally, the maximum circumferential stress criterion, where just the phonon stress is supposed to be relevant for fracture processes, predicts a minor impact of phonon-phason coupling on the crack deflection angle. The J-integral criterion, where the total potential energy is considered, postulates crack propagation into the direction going along with the maximal reduction of phonon and phason energies. Accordingly, the impact of field-coupling on crack deflection may be nonnegligible. Work in progress deals with crack path simulations. First results indicate that the curvature of crack paths in a 1D QC may be augmented or reduced depending on the direction of the quasicrystalline axis and the magnitudes of the coupling coefficients. 
A Material constants and characteristic matrices

In compressed notation the constitutive equations of a $1 \mathrm{D}$ QC with the QA being the $x_{1}$-axis are

$$
\left(\begin{array}{l}
\sigma_{11} \\
\sigma_{22} \\
\sigma_{12} \\
H_{11} \\
H_{12}
\end{array}\right)=\left(\begin{array}{ccccc}
C_{1111} & C_{1122} & 0 & R_{1} & 0 \\
C_{1122} & C_{2222} & 0 & R_{2} & 0 \\
0 & 0 & C_{1212} & 0 & R_{3} \\
R_{1} & R_{2} & 0 & K_{1} & 0 \\
0 & 0 & R_{3} & 0 & K_{2}
\end{array}\right)\left(\begin{array}{c}
\varepsilon_{11} \\
\varepsilon_{22} \\
2 \varepsilon_{12} \\
w_{11} \\
w_{12}
\end{array}\right)
$$

The following material constants have been used for the calculations [46, 47, 48]:

$$
\begin{aligned}
& C_{1111}=232.22, \quad C_{1122}=66.63, \quad C_{2222}=234.33 \\
& C_{1133}=66.63, \quad C_{2233}=57.41, \quad C_{3333}=234.33, \\
& C_{1212}=70.19, \quad C_{1313}=70.19, \quad C_{2323}=88.46, \\
& R_{1}=-1.1, \quad R_{2}=0.2, \quad R_{3}=0.5 \\
& K_{1}=122, \quad K_{2}=24,
\end{aligned}
$$

where the unit is GPa. For plane stress conditions, the following transformations are required:

$$
\begin{aligned}
& C_{1111}^{s}=C_{1111}-\frac{C_{1133} C_{1133}}{C_{3333}}, \\
& C_{1122}^{s}=C_{1122}-\frac{C_{2233} C_{1133}}{C_{3333}}, \\
& C_{2211}^{s}=C_{1122}^{s}, \quad C_{2222}^{s}=C_{2222}-\frac{C_{2233} C_{2233}}{C_{3333}} \\
& C_{1212}^{s}=C_{1212}, \\
& R_{1}^{s}=R_{1}-\frac{C_{2233} R_{1}}{C_{3333}}, \quad R_{2}^{s}=R_{2}-\frac{C_{1133} R_{1}}{C_{3333}} \\
& R_{3}^{s}=R_{3}, \quad \\
& K_{1}^{s}=K_{1}-\frac{R_{1} R_{1}}{C_{3333}}, \quad K_{2}^{s}=K_{2} .
\end{aligned}
$$

The superscript index $s$ indicates the constants for plane stress conditions.

In the general case, the characteristic matrices are $6 \times 6$, where 3 of them are from the phonon and the other 3 from the phason field. However, for a plane 1D QC, only 2 of the phonon and 1 of the phason dimensions are required. So, for the quasiperiodic direction along $x_{1}$ the following complex $3 \times 3$ and $1 \times 3$ matrices are calculated applying the methods introduced in $[38]$ :

$$
\begin{aligned}
& A_{M N}=\left(\begin{array}{ccc}
-0.00858494 & 1.13626 & 0.55096 i \\
-0.00293218 i & 0.539528 i & -1.14174 \\
0.999967 & 0.00337372 & -0.00374684 i
\end{array}\right), \\
& B_{M N}=\left(\begin{array}{ccc}
-0.4371 i & 148.1 i & -107.7 \\
0.1939 & -107.2 & -151.3 i \\
54.10 i & 1.167 i & -0.7029
\end{array}\right) \times 10^{9}, \\
& p_{N}=(2.25472 i, 1.38257 i, 0.711723 i) .
\end{aligned}
$$

For the quasiperiodic direction along $x_{2}$ they are

$$
\begin{aligned}
& A_{M N}=\left(\begin{array}{ccc}
-1.14174 & 0.539528 i & -0.00293218 i \\
-0.55096 i & -1.13626 & 0.00858494 \\
0.00374684 i & -0.00337372 & -0.999967
\end{array}\right), \\
& B_{M N}=\left(\begin{array}{ccc}
-151.3 i & -107.1 & 0.1939 \\
107.7 & -148.1 i & 0.4371 i \\
0.7029 & -1.167 i & -54.10 i
\end{array}\right) \times 10^{9}, \\
& p_{N}=(1.40504 i, 0.723288 i, 0.443514 i) .
\end{aligned}
$$

The matrices $P_{M N}^{\mathrm{S} 1}, P_{M N}^{\mathrm{S} 2}, P_{M N}^{\mathrm{u}}$ are diagonal matrices and functions of the polar coordinate angle $\theta$ :

$$
\begin{aligned}
& P_{M N}^{\mathrm{S} 1}=\operatorname{diag}\left(\frac{p_{J}}{\sqrt{\cos (\theta)+p_{J} \sin (\theta)}}\right), \\
& P_{M N}^{\mathrm{S} 2}=\operatorname{diag}\left(\frac{1}{\sqrt{\cos (\theta)+p_{J} \sin (\theta)}}\right), \\
& P_{M N}^{\mathrm{u}}=\operatorname{diag}\left(\sqrt{\cos (\theta)+p_{J} \sin (\theta)}\right) .
\end{aligned}
$$

Eq. (18) shows the analytical solution near the crack tip. Alternatively, the stresses and displacements can be split into parts, introducing angular functions being independent on geometry and loading. In detail, the equations of a 3D QC are given as

$$
\begin{gathered}
\sigma_{M j}=\frac{1}{\sqrt{2 \pi r}}\left(K_{I I}^{\|} f_{M j}^{\| I I}+K_{I}^{\|} f_{M j}^{\| I}+K_{I I I}^{\|} f_{M j}^{\| I I I}+K_{I I}^{\perp} f_{M j}^{\perp I I}\right. \\
\left.+K_{I}^{\perp} f_{M j}^{\perp I}+K_{I I I}^{\perp} f_{M j}^{\perp I I I}\right), \\
u_{M}=\sqrt{\frac{2 r}{\pi}}\left(K_{I I}^{\|} d_{M}^{\| I I}+K_{I}^{\|} d_{M}^{\| I}+K_{I I I}^{\|} d_{M}^{\| I I I}+K_{I I}^{\perp} d_{M}^{\perp I I}\right. \\
\left.+K_{I}^{\perp} d_{M}^{\perp I}+K_{I I I}^{\perp} d_{M}^{\perp I I I}\right),
\end{gathered}
$$

where $f_{M j}^{J}$ are the angular functions of stresses and $d_{M j}^{J}$ those of the displacements. For plane problems $j=1,2$, so the angular functions are

$$
\begin{aligned}
f_{M 1}^{J} & =-\Re\left\{B_{M N} P_{N I}^{\mathrm{S} 1} B_{I J}^{-1}\right\}, \\
f_{M 2}^{J} & \left.=\Re\left\{B_{M N} P_{N I}^{\mathrm{S} 2} B_{I J}^{-1}\right]\right\}, \\
d_{M}^{J} & \left.=\Re\left\{A_{M N} P_{N I}^{\mathrm{u}} B_{I J}^{-1}\right]\right\} .
\end{aligned}
$$

The index $J$ of the angular functions runs from 1 to 6 and denotes the corresponding stress intensity factor.

\section{References}

1. D. Shechtman, I. Blech, D. Gratias, J.W. Cahn, Phys. Rev. Lett. 53, 1951 (1984)

2. Pauling, Phys. Rev. Lett. 58(4), 365 (1987)

3. D. Levine, P.J. Steinhardt, Phys. Rev. Lett. 53, 2477 (1984)

4. P. Bak, Phys. Rev. Lett. 54(14), 1517 (1985)

5. W. Steurer, S. Deloudi, Crystallography of Quasicrystals: Concepts, Methods and Structures, Springer series in materials science, vol. 126 (Springer-Verlag Berlin Heidelberg, Berlin, Heidelberg, 2009)

6. L.X. He, Y.K. Wu, K. Kuo, J. Mater. Sci. Lett. 7(12), 1284 (1988)

7. B. Dubost, J.M. Lang, M. Tanaka, P. Sainfort, M. Audier, Nature 324(6092), 48 (1986) 
8. L. Bindi, P.J. Steinhardt, N. Yao, P.J. Lu, Science (New York, N.Y.) 324(5932), 1306 (2009)

9. W. Steurer, Z. Kristallogr. 219(7-2004), 391 (2004)

10. M. Cardona, P. Fulde, K.v. Klitzing, R. Merlin, H.J. Queisser, H. Störmer, Z.M. Stadnik (eds.), Physical Properties of Quasicrystals. Springer Series in Solid-State Sciences (Springer Berlin Heidelberg, Berlin, Heidelberg, 1999)

11. P. Gong, C.Z. Hu, X. Zhou, L. Miao, X. Wang, Eur. Phys. J. B 52(4), 477 (2006)

12. T. Fujiwara, Mater. Sci. Forum 150-151, 491 (1994)

13. C. Malgrange, C. Ricolleau, M. Schlenker, Symmetry and Physical Properties of Crystals (Springer Netherlands, Dordrecht, 2014)

14. S. Takeuchi, Mater. Sci. Forum 150-151, 35 (1994)

15. W. Shi, Appl. Math. Comput. 215(3), 1062 (2009)

16. Y. Gao, A. Ricoeur, Philos. Mag. 92(34), 4334 (2012)

17. X. Wang, P. Schiavone, Mathematics and Mechanics of Complex Systems 1(1), 1 (2013)

18. E. Pan, Electronic Journal of Boundary Elements 1(2), 236 (2003)

19. Y. Gao, A. Ricoeur, P. Roy. Soc. A-Math. Phy. 467(2133), $2622(2011)$

20. X.F. Li, T.Y. Fan, Y.F. Sun, Philos. Mag. A 79(8), 1943 (1999)

21. Y. Gao, A. Ricoeur, L. Zhang, Phys. Lett. A 375(28-29), $2775(2011)$

22. J. Sladek, V. Sladek, S.N. Atluri, Eng. Fract. Mech. 140, $61(2015)$

23. L.Z. Yang, A. Ricoeur, F.M. He, Y. Gao, Chinese Phys. B 23(5) (2014)

24. R. Mikulla, J. Stadler, F. Krul, H.R. Trebin, P. Gumbsch, Phys. Rev. Lett. 81(15), 3163 (1998)

25. P. Bak, Phys. Rev. Lett. 56(8), 861 (1986)

26. T. Janssen, Acta Crystallogr. A 42(4), 261 (1986)

27. C. Janot, Quasicrystals: A primer, Monographs on the physics and chemistry of materials, vol. 50, 2nd edn. (Clarendon Press and Oxford University Press, Oxford and New York, 1994)

28. I.R. Fisher, Z. Islam, A.F. Panchula, K.O. Cheon, M.J. Kramer, P.C. Canfield, A.I. Goldman, Philos. Mag. B $77(6), 1601$ (1998)

29. K. Nagao, T. Inuzuka, K. Nishimoto, K. Edagawa, Phys. Rev. Lett. 115(7) (2015)

30. D. Ding, W. Yang, C. Hu, R. Wang, Mater. Sci Forum 150-151, 345 (1994)

31. J. Socolar, T. Lubensky, P. Steinhardt, Phys. Rev. B 34(5), 3345 (1986)

32. M. de Boissieu, Chem. Soc. Rev. 41(20), 6778 (2012)

33. D. Levine, T. Lubensky, S. Ostlund, S. Ramaswamy, P. Steinhardt, J. Toner, Phys. Rev. Lett. 54(14), 1520 (1985)

34. D. Ding, W. Yang, C. Hu, R. Wang, Phys. Rev. B 48(10), $7003(1993)$

35. C. Hu, R. Wang, D. Ding, Rep. Prog. Phys. 63(1), 1 (2000)

36. M. Feuerbacher, C. Metzmacher, M. Wollgarten, K. Urban, B. Baufeld, M. Bartsch, U. Messerschmidt, Mat. Sci. Eng. A-Struct. 233(1), 103 (1997)

37. T. Fan, Mathematical Theory of Elasticity of Quasicrystals and Its Applications (Springer-Verlag Berlin Heidelberg, Berlin, Heidelberg, 2011)

38. Y. Gao, A. Ricoeur, L.L. Zhang, L.Z. Yang, Arch. Appl. Mech. 84(8), 1103 (2014)

39. M. Kuna, Finite Elements in Fracture Mechanics, Solid Mechanics and Its Applications, vol. 201 (Springer Netherlands, 2013)

40. H. Ma, Y.H. Chen, Int. J. Fracture 75(2), R25 (1996)
41. P.O. Judt, A. Ricoeur, G. Linek, Eng. Fract. Mech. 138, 33 (2015)

42. J.D. Eshelby, Philos. T. Roy. Soc. A 244(877), 87 (1951)

43. F. Erdogan, G.C. Sih, J. Basic Eng.-T ASME 85(4), 519 (1963)

44. P.O. Judt, A. Ricoeur, Int. J. Fracture 182(1), 53 (2013)

45. U. Koschella, F. Gähler, J. Roth, H.R. Trebin, J. Alloy Compd. 342(1-2), 287 (2002)

46. M.A. Chernikov, H.R. Ott, A. Bianchi, A. Migliori, T.W. Darling, Phys. Rev. Lett. 80(2), 321 (1998)

47. H.C. Jeong, P.J. Steinhardt, Phys. Rev. B 48(13), 9394 (1993)

48. K. Edagawa, Philos. Mag. 87(18-21), 2789 (2007) 